\title{
Almacenamiento de carbono en especies predominantes de flora en el lago Chinchaycocha
}

\author{
Carbon storage in predominant species of flora in Chinchaycocha lake
}

Ronald Medrano Yanqui', Liz Chupan Minaya², Miguel Vila Balbín 3 . Universidad Continental

\section{RESUMEN}

Objetivos: Evaluar la cantidad de carbono que almacenan las especies predominantes de flora del lago Chinchaycocha. Métodos: La investigación fue de tipo básica, de corte transversal y con un nivel exploratorio-comparativo. El estudio se hizo en tres ecosistemas dentro del humedal: bofedal, pajonal y totoral, las áreas fueron identificadas con ayuda de imágenes satelitales Landsat. El procedimiento estuvo basado en la recolección de muestras divididas en: biomasa aérea, biomasa radicular y muestras de suelo, hallándose el contenido de carbono de cada una. Resultados: En el totoral se obtuvo que Schoenoplectus californicus Var. Tatora almacena $30,65 \mathrm{tC} /$ ha y Juncos arcticus Var. Andicola 8,70 +C/ha. En el pajonal Deyeuxia recta Kunth almacena $7,02 \mathrm{tC} /$ ha en su biomasa aérea y $8,41 \mathrm{tC} /$ ha en su biomasa radicular. En el bofedal: Plantago tubulosa almacena $0,81+\mathrm{C} / \mathrm{ha}$ en su biomasa aérea y $1,88+\mathrm{C} /$ ha en su biomasa radicular, Eleocharis albibracteata almacena 0,22 † $\mathrm{C} /$ ha en su biomasa aérea y 2,95 †C/ha en su biomasa radicular, y Limosella australis almacena $0,22 \mathrm{tC} /$ ha en su biomasa aérea y $0,38 \mathrm{tC} / \mathrm{ha}$ en su biomasa radicular. En el almacenamiento de carbono en suelos se determinó 774,76 †C/ ha en pajonales y $684,58 \mathrm{tC} / \mathrm{ha}$ en bofedales. Conclusiones: Se concluye que en el lago Chinchaycocha el ecosistema que brinda mayor almacenamiento de carbono es el totoral, seguido por el pajonal y en menor proporción el bofedal. Asimismo los suelos son considerados los mayores sumideros de carbono.

1 Ingeniero Químico, jefe de la Reserva Nacional de Junín, investigador de la Universidad Continental

2 Bachiller en Ing. Forestal y del Ambiente, investigadora de la Universidad Continental

3 Bachiller en Ing. Forestal y del Ambiente, investigador de la Universidad Continental

Palabras clave: Almacenamiento de carbono, flora, humedales, lago Chinchaycocha.

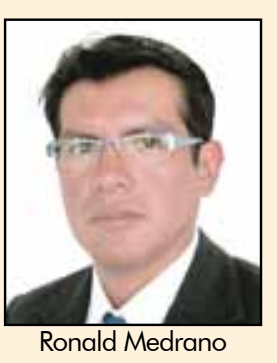

ronald_medrano@hotmail.com 


\section{ABSTRACT}

Objectives: Assess the amount of carbon storage of the predominant species of flora in the Chinchaycocha lake. Methods: The type research was basic, cross-sectional and with a exploratory-comparative level. The study was done in three ecosystems within the lake: wetland, grassland and cattails, which were identified using Landsat satellite images. The procedure was based on the collection of samples divided into: aboveground biomass, root biomass and soil samples and then determining the carbon content of each one. Results: In the cattails it was obtained that Schoenoplectus californicus Var. Tatora stores $30.65 \mathrm{tC} / \mathrm{ha}$ and Juncos arcticus Var. Andicola stores 8,70 tC/ha. In the grassland Deyeuxia recta Kunth stores $7,02+\mathrm{tC} / \mathrm{ha}$ in its aboveground biomass and 8,41 tC/ha in its root biomass. In the wetland Plantago tubulosa stores $0,81 \mathrm{tC} / \mathrm{ha}$ in its aboveground biomass and $1,88 \mathrm{tC} / \mathrm{ha}$ in its root biomass. Eleocharis albibracteata stores $0,22 \mathrm{tC} / \mathrm{ha}$ in its aboveground biomass and 2,95 $\mathrm{tC} / \mathrm{ha}$ in its root biomass, and Limosella australis stores $0,22 \mathrm{tC} / \mathrm{ha}$ in its aboveground biomass and $0,38 \mathrm{tC} / \mathrm{ha}$ in its root biomass. The soil carbon storage was determined for $774,76 \mathrm{tC} / \mathrm{ha}$ in grassland and $684,58 \mathrm{tC} /$ ha in wetland. Conclusions: We concluded that the ecosystem of Chinchaycocha lake whit the highest amount of carbon storage is cattails, followed by grasslands and to a lesser extent the wetlands. Also, the soils are considered the biggest carbon sinks.

Keywords: Carbon storage, flora, wetlands, Chinchaycocha Lake.

\section{INTRODUCCIÓN}

Se sabe que los bosques son los mayores sumideros de carbono, pero cuánto carbono son capaces de almacenar las especies de flora de un humedal, es una pregunta que el proyecto buscó responder. El dióxido de carbono es uno de los gases más importantes cuando se habla de calentamiento global, entonces el buscar soluciones para mitigar esta situación nos lleva a preguntarnos, qué importancia tienen aquellos ecosistemas pocos mencionados en la solución, así es el caso de los humedales, ecosistemas capaces de almacenar grandes cantidades de carbono gracias a la acción fotosintética de la flora (proceso por el cual el dióxido de carbono se toma de la atmósfera y que en el interior de la planta es transformado en proteínas, carbohidratos, etc) y a los procesos lentos de descomposición de la materia muerta que ocurren en el suelo, ayudando a la retención de estos gases por más tiempo.

Con el aumento de la población, se han incrementado las amenazas para los humedales y su desaparición implica la extinción de sus funciones ecológicas (1); en tanto el lago Chinchaycocha considerado el segundo lago mas importante en el Perú después del lago Titicaca, protegido mediante el establecimiento de la Reserva Nacional de Junín (2); está deteriorándose por las acciones humanas, fundamentalmente por la contaminación de los relaves mineros cuyas descargas a los ríos desembocan en este humedal, el mal manejo de los niveles de embalse y desembalse (3), la extracción de la flora para uso doméstico y la quema indiscriminada de totorales; debido al desconocimiento del valor de sus beneficios ambientales como uno de los ecosistemas más frágiles (4) y a la vez productivos de la tierra (5).

En el presente estudio se determinó el almacenamiento de carbono de las especies predominantes del bofedal, pajonal y totoral del lago Chinchaycocha, para lo cual se consideró el estudio de la flora predominante y el suelo debido a que este último representa una de las fuentes con mayor reserva en el planeta, ya que si los humedales se aprovechan con responsabilidad pueden representar beneficios para la sociedad. 


\section{MATERIAL Y METODOS}

Se usó el método de observación y el analítico, ya que los resultados fueron por estudios en campo teniendo como premisa alcances concretos sobre el tema y se analizó los datos obtenidos en el campo y laboratorio; fue de tipo básica, debido a que ayuda a generar conocimientos, sobre la cantidad de carbono que almacenan las especies predominantes de flora en totorales, bofedales y pajonales del lago Chinchaycocha; es de corte transversal con un nivel exploratorio - comparativo.

La población total comprende tres ecosistemas: bofedales, pajonales $y$ totorales con una extensión total de 33566,41 ha. La distribución de estos ecosistemas se muestra en la Figura № 1.

Para los estratos de bofedales y pajonales la distribución de parcela a evaluar fue siguiendo el método Cluster (6), donde se trata de abarcar la mayor área representativa de la población, evaluándose 20 parcelas puntuales para pajonales y 15 parcelas puntuales para los bofedales. Para los totorales, el muestreo fue dirigido, evaluándose un total de 31 parcelas puntuales. Para la recolección de muestras se tomaron en cuenta a tres especies de flora en el bofedal, una especie en el pajonal y dos especies en el totoral, el estudio consistió en la extracción de la biomasa aérea y biomasa radicular de cada especie en estudio, para ello fue necesaria la selección de muestras, con excepción para la flora de totorales por la poca accesibilidad de las áreas; también se extrajo muestras de suelo hasta $20 \mathrm{~cm}$ de profundidad (bofedales y pajonales). Los análisis químicos para flora fueron realizados en el Laboratorio de Suelo de la Universidad Nacional Agraria La Molina (UNALM) y para suelo en el Laboratorio de Suelos del Instituto Nacional de Innovación Agraria (INIA). Los datos fueron trabajados determinándose el almacenamiento de carbono para cada especie en estudio.

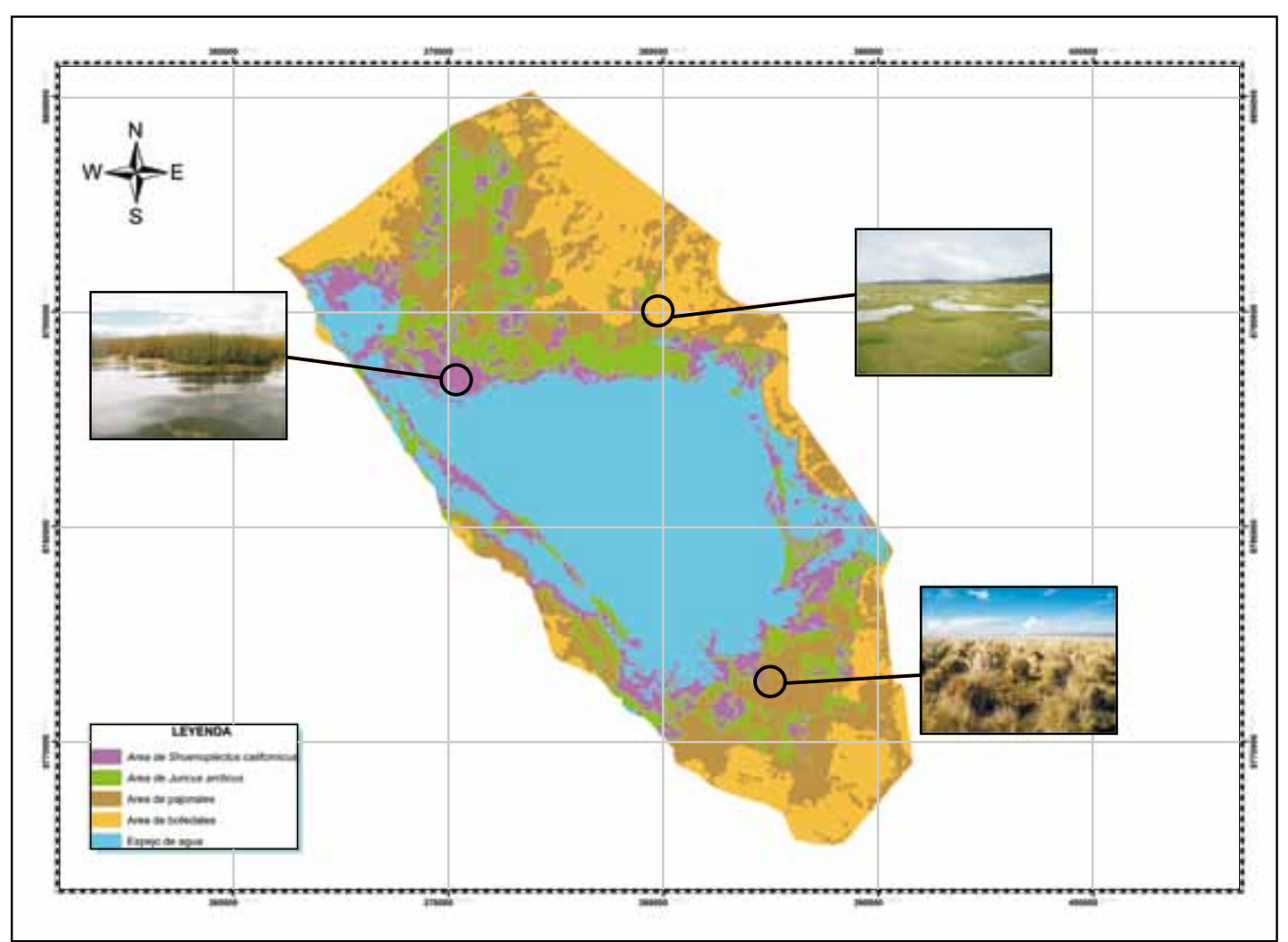

Figura 1: Delimitación de áreas de bofedales, pajonales y totorales. 
El cálculo del almacenamiento de carbono fue de la siguiente manera:

\section{Determinación del carbono en biomasa aérea y radicular}

a. Materia seca

$M S=(p s m / p f m)^{*} p f t$

Donde:

MS = Materia seca del componente

psm = Peso seco de la muestra

pfm = Peso fresco de la muestra

$\mathrm{Pft}=$ Peso fresco total del componente

b. Carbono

$\mathrm{C}=\mathrm{MS} \times \mathrm{Fc}$

Donde:

$\mathrm{C}=$ Carbono capturado $\mathrm{C} / \mathrm{ha}$ MS = Materia seca del componente

Fc $=$ Factor de conversión (obtenido del método Walkey y Black)

c. Transformación a toneladas por hectárea

Los resultados obtenidos fueron dados en $\mathrm{kg} / \mathrm{m} 2$, por tal motivo para determinar el carbono en toneladas por hectárea se hizo una simple conversión de unidades.

\section{Determinación del carbono en el suelo}

a. Porcentaje de carbono

$\% \mathrm{C}=\% \mathrm{M} . \mathrm{O} / 1,724$

Donde:

$\% \mathrm{M} . \mathrm{O}=$ Porcentaje de materia orgánica (obtenido del método Walkey y Black).

MS = 1,724 (factor constante)

$\% \mathrm{C}=$ Porcentaje de Carbono

b. Peso del suelo

$\mathrm{Ps}=\mathrm{V} \times \mathrm{Dap}$

Donde:

Ps $=$ Peso del suelo

$\mathrm{V} \quad=$ Volumen del suelo

Dap $=$ Densidad aparente del suelo

c. Carbono total

$C=P s \times \% C$

Donde:

$\mathrm{C}=$ Carbono total capturado $+\mathrm{C} /$ ha

Ps $=$ Peso del suelo

$\%$ C $=$ Porcentaje de Carbono

Tabla № 1: Relación de especies en estudio según ecosistemas.

\begin{tabular}{lccc}
\hline \multicolumn{1}{c}{$\begin{array}{c}\text { Especies en } \\
\text { estudio }\end{array}$} & Bofedales & Ecosistemas & Totorales \\
\hline $\begin{array}{l}\text { Plantago tubulosa } \\
\text { Eleocharis albibracteata }\end{array}$ & $\mathrm{x}$ & $\mathrm{x}$ & $\mathrm{x}$ \\
Limosella australis & $\mathrm{x}$ & \\
Deyeuxia recta Kunth & & $\mathrm{x}$ \\
Schoenoplectus californicus Var. Tatora & & \\
Juncos arcticus Var. Andicola & & \\
\hline
\end{tabular}




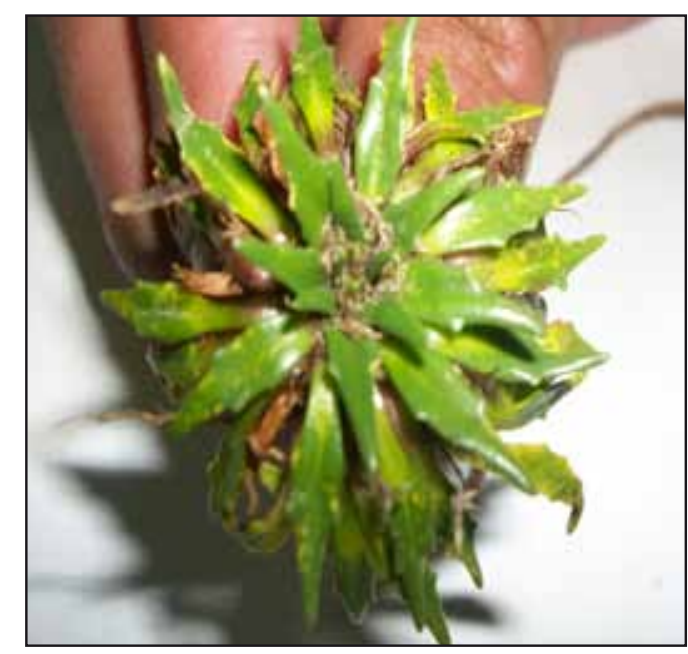

Plantago tubulosa

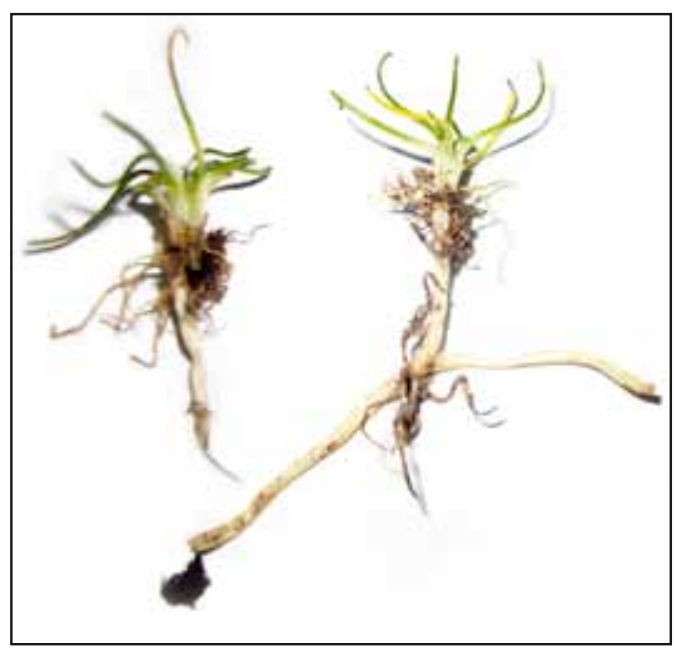

Limosella australis

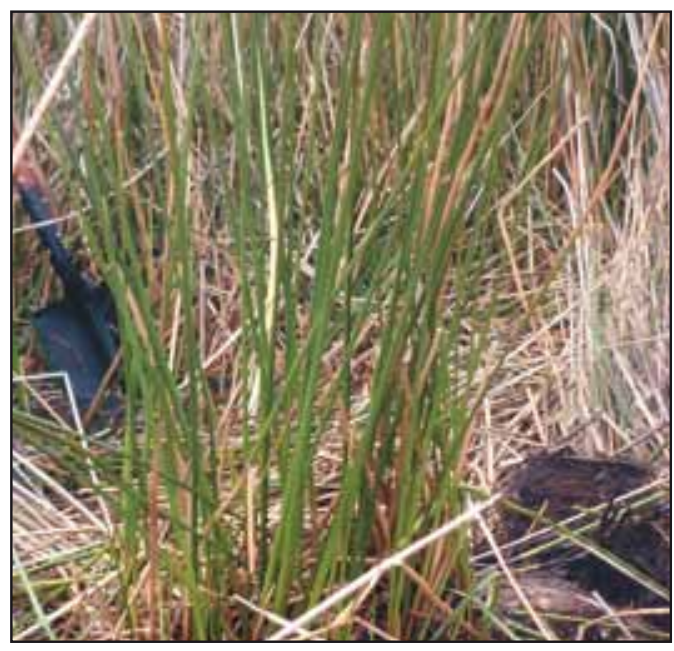

Juncos arcticus Var. Andicola

Figura $\mathrm{N}^{0} 2$ : Especies de flora estudiadas.

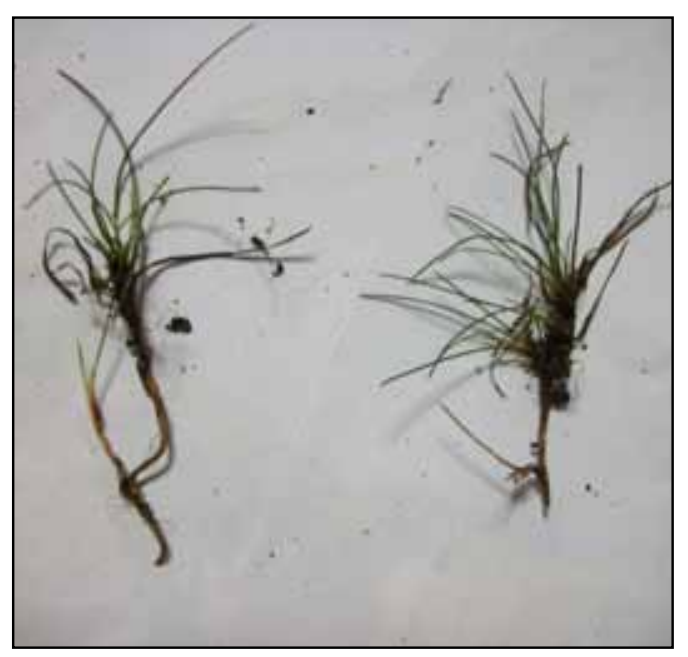

Eleocharis albibracteata

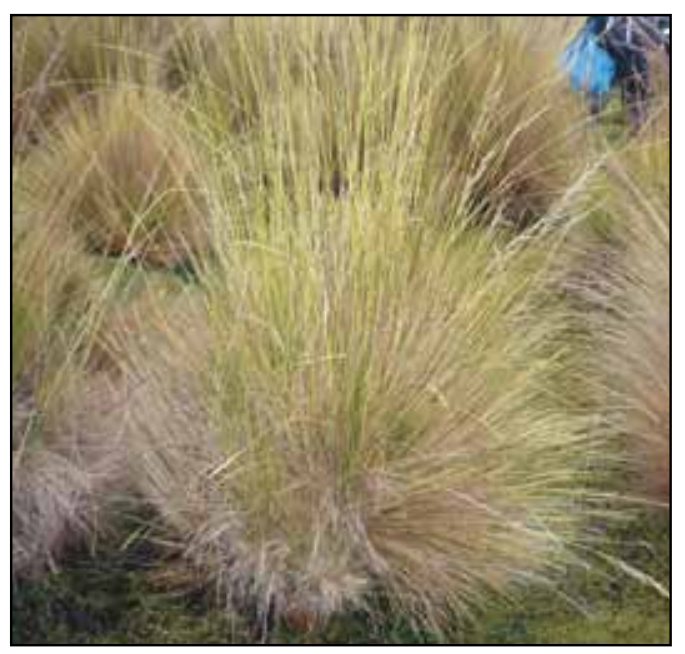

Deyeuxia recta Kunth

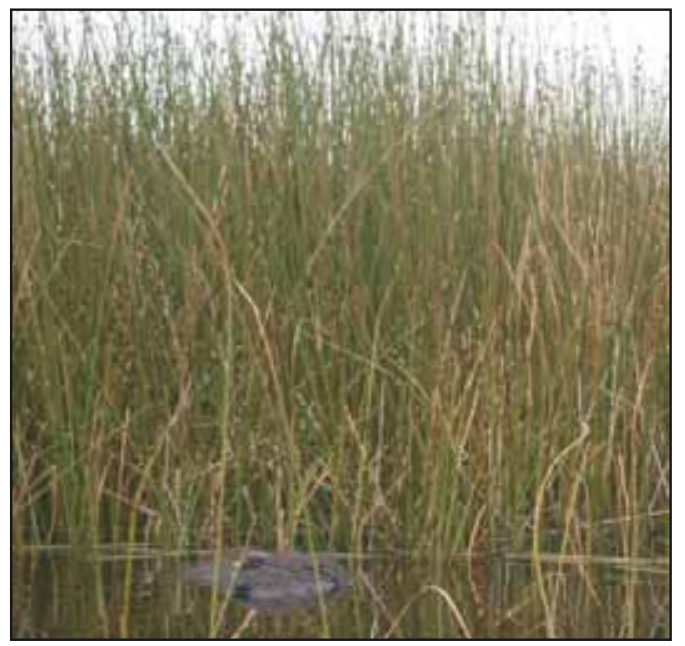

Schoenoplectus californicus Var. Tatora

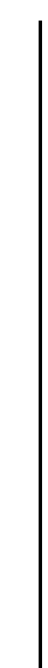




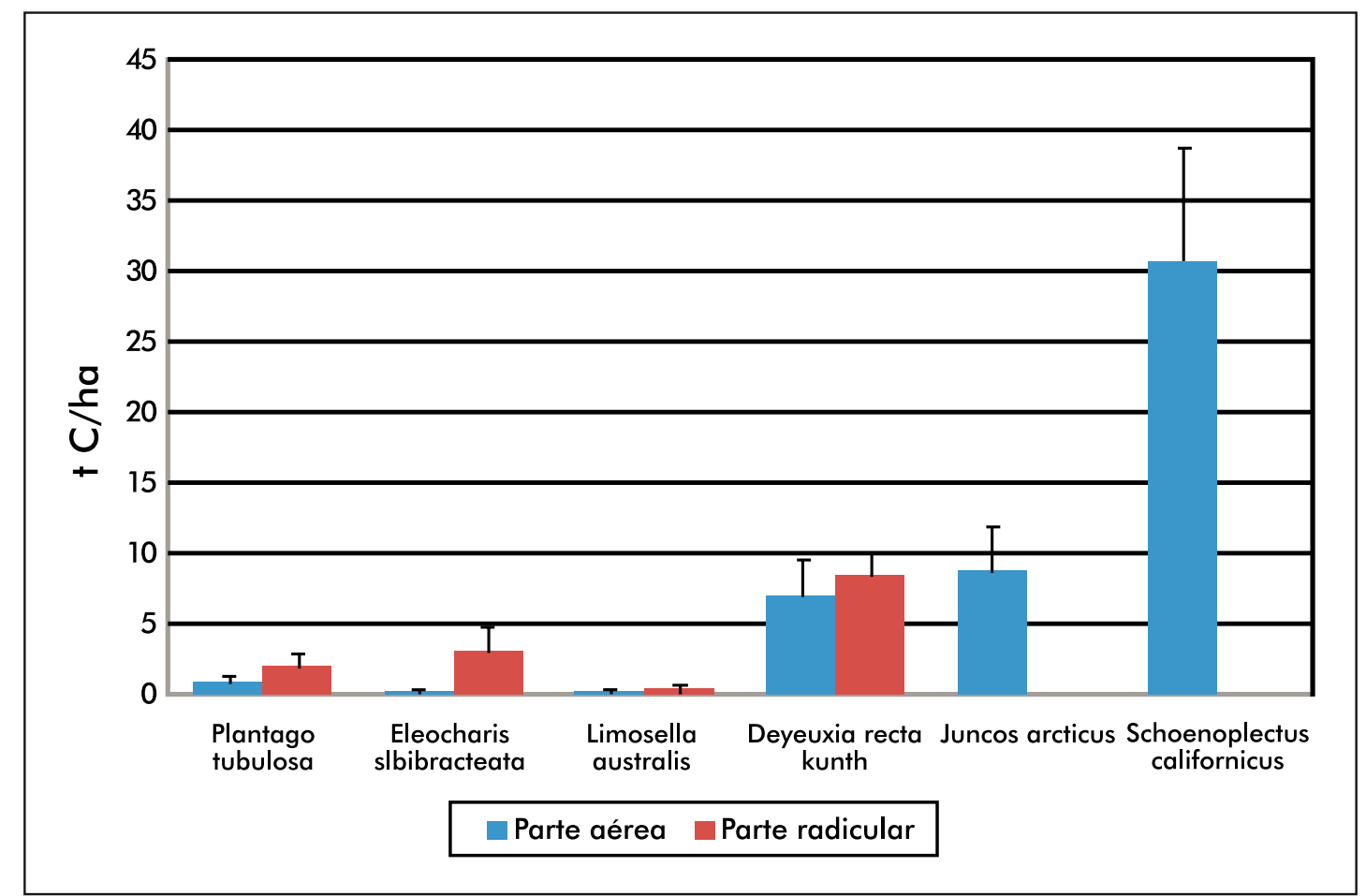

Figura $N^{\circ}$ 3: Carbono almacenado en la flora del lago Chinchaycocha.

\section{RESULTADOS}

En la Tabla № 1 se presentan las especies predominantes del lago Chinchaycocha, las cuales fueron seleccionadas tomando en cuenta la abundancia de estas especies para cada ecosistema dentro del área de estudio.

Tabla № 2: Porcentaje de materia orgánica en el suelo.

\begin{tabular}{lc}
\hline Muestra & $\%$ MO \\
\hline Bofedal & 39,34 \\
Pajonal & 47,70 \\
Promedio & 43,52 \\
\hline
\end{tabular}

Se puede inferir que las especies del ecosistema totoral presentan mayor dispersión, debido a que estas especies llegan a medir hasta 4 metros (en el caso de Schoenoplectus californicus Var. Tatora) y por tanto el carbono almacenado es mayor en comparación con las especies de los otros ecosistemas, donde prevalecen especies rastreras (en el caso del ecosistema bofedal) (Figura $\mathrm{N}^{\circ} 3$ ).

Se observa que la mayor concentración de materia orgánica está en el suelo del ecosistema pajonal (Tabla $\mathrm{N}^{\circ} 2$ ), esto se

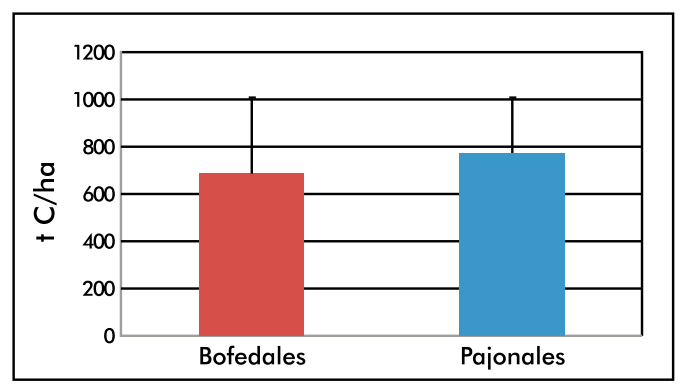

FiguraN:Capturadecarbonoenlossuelosdelhumedal

debe a que estos suelos se encuentran en zonas cercanas a los totorales por tanto son áreas pantanosas donde la descomposición de la materia orgánica es más lenta en comparación con los suelos del ecosistema bofedal.

La estructura radicular almacena más carbono que la estructura aérea tanto para el ecosistema bofedal como para el 
ecosistema pajonal, esto se debe a que en la raíz se almacena los carbohidratos, proteínas, etc, que son productos del proceso fotosintético.

El carbono almacenado en los suelos del ecosistema pajonal es mayor en comparación con el carbono almacenado en los suelos del ecosistema bofedal (Figura $N^{\circ} 4$ ), además se infiere que el carbono almacenado en los suelos del ecosistema bofedal presentan mayor dispersión, esto debido a que en algunas zonas los bofedales permanecen inundadas y en otras zonas son áreas secas; mientras que los pajonales dentro del área de estudio se encuentran en zonas anegadas o pantanosas donde el carbono es almacenado por más tiempo, encontrándose valores altos.

\section{DISCUSIÓN}

Con respecto a la especie de totora, Schoenoplectus californicus Var. Tatora, parte aérea, se ha determinado que esta especie captura 30,65 † C/ha, en comparación con Palomino (7) que da como resultado 20,1 + C/ha. En un estudio realizado en el humedal de Puerto Viejo - Lima, esto pudo darse debido a que las totoras en el lago Chinchaycocha tuvieron mayor altura entre $3-4 \mathrm{~m}$.

En el análisis de suelos para bofedales se reporta 39,34 \% de materia orgánica, mayor a la reportada en un estudio realizado por Miranda (8) en el bofedal artificial de Quinsanchata en Santa Lucia que presenta datos de un $0,9 \%-5,76 \%$ de materia orgánica en los suelos de los bofedales, esta diferencia se debe a que Quinsanchata es un bofedal artificial y Chinchaycocha un bofedal natural que se formó hace muchos años.

En un promedio final del análisis de los suelos: bofedales y pajonales, permite establecer una concentración de materia organica de $43,52 \%$, observándose valores más altos en humedales de la selva, así como reporta Freitas L, et al (9) en la Reserva Nacional de Pacaya Samiria cuyos valores alcanzan a $75,13 \%$ en el aguajal denso, y $76,81 \%$ en el aguajal mixto. Asimismo Moreno, et al (10) reporta que en los suelos de manglares de Tabasco los contenidos de materia orgánica son muy altos, llegando hasta $48,3 \%$ en suelos donde se ubica el mangle rojo y $85,7 \%$ en suelos del mangle blanco, con esto se reafirma lo que dicen Kilham y Alexander (11), que hay mayor cantidad de materia orgánica en suelos que permanecen inundados durante mucho tiempo y con aportes constantes de materia orgánica.

Para los suelos de los bofedales del estudio realizado se tuvo una fijación de 684,58 † $\mathrm{C} /$ ha. Castro $\mathrm{M}$. reporta para un estudio ejecutado en los bofedales de Oña Nabon - Saraguro - Yacuambi una fijación de carbono en los suelos de 889,36 + C/ha (12), al mismo tiempo en los bofedales del Frente Suroccidental de Tungurahua establece para los suelos 519,15 + C/ha, estos bofedales representan los páramos ecuatorianos; es notable la diferencia que existen entre estos tres bofedales.

Castro en sus resultados menciona la importancia que brindan estos suelos si se encuentran en forma natural y sin intervención humana; los bofedales de Oña - Nabon - Saraguro - Yacuambi son bofedales naturales mientras que los del Frente Suroccidental de Tungurahua vienen siendo afectados por la agricultura y ganadería, al igual que los bofedales del Lago Chinchaycocha en donde se viene dando el sobrepastoreo, contaminación y extracción irracional de pastos.

Dentro del lago Chinchaycocha el suelo representa un importante sumidero de carbono, ya que en su interior fija 729,67 † $\mathrm{C} /$ ha en promedio, demostrándose así que el carbono orgánico del suelo es 2,7 veces más que la reserva biótica (13).

\section{Agradecimiento}

A la Reserva Nacional de Junín por el apoyo en la ejecución de esta investigación. 


\section{REFERENCIAS BIBLIOGRÁFICAS}

1 Grupo de expertos de la evaluación de los ecosistemas del milenio. Los ecosistemas y el bienestar humano: Humedales y agua. Informe de síntesis. Washington: World Resources Institute; 2005. Serie de Informe síntesis: QH541.5.M3E275.

2 Intendencia de Áreas Naturales Protegidas. Plan Maestro de la Reserva Nacional de Junín 20082012 - Documento de trabajo [Internet]. Lima: Instituto Nacional de Recursos Naturales; 2009 [citado 08/09/11]. Disponible en: http://www. sernanp.gob.pe/sernanp/archivos/ biblioteca/publicaciones/RN_Junin/ PlanMaestro2008-2012-RNJunin.pdf

3 CESEL Ingenieros. Informe de simulación de calidad de aire: Plan de manejo ambiental de las operaciones de embalse y desembalse del lago Chinchaycocha. Lima: Electroperu; 2007. Informe final: 8.

4 Aponte H, Ramírez D. Humedales de la costa central del Perú: Estructura y amenazas de sus comunidades vegetales. Ecología Aplicada. 2011; 10(1): 31-39.

5 Hernández M. Suelos de Humedales como sumideros de carbono y fuentes de Metano. Terra Latinoamericana. 2010; 28(2): 139-147.

6 Viceministerio de desarrollo estratégico de recursos naturales. Identificación de metodologías existentes para determinar stock de carbono en ecosistemas forestales - Documento de trabajo [Internet]. Lima: Ministerio Nacional del Ambiente; 2009 [citado 10/11/11]. Disponible en: http:// redpeia.minam.gob.pe/admin/files/ item/4d77f55b028f2_Metodologias_ para_Stock_de_Carbono.pdf

7 Palomino D. Estimación del servicio ambiental de captura del $\mathrm{CO} 2$ en la flora de los humedales de Puerto Viejo (Tesis para optar el grado de Magíster en Ciencias Ambientales). Lima:
Universidad Mayor de San Marcos; 2007.

8 Miranda F. Evaluación AgrostoEdafológica de los pastizales del centro experimental de Quinsachata (Tesis para optar el grado de Ingeniero Agrónomo). Puno: Universidad Nacional del Altiplano; 1989.

9 Freitas L, Otárola E, Del Castillo D, Linares C, Martínez P, Malca. Servicios ambientales de almacenamiento y secuestro de carbono del ecosistema aguajal en la Reserva Nacional Pacaya Samiria-Loreto. Lima: Instituto de Investigación de la Amazonia Peruana; 2006. Documento técnico №29.

10 Moreno E, Guerrero A, Gutiérrez $M$, Ortiz A, Palma J. Los manglares de Tabasco, una reserva natural de carbono. Madera y bosques. 2002; 8 (1): 115-128.

11 Kilham O, Alexander M. A basis for organic matter accumulation in soil under anaerobiosis. Soil Sciencia. 1984; 137 (6): 419-427.

12 Castro, M. Una valoración económica del almacenamiento de agua y carbono en los bofedales de los páramos ecuatorianos - la experiencia en Oña - Nabón - Saraguro - Yacuambi y el Frente Suroccidental de Tungurahua. EcoCiencia / Wetlands International / UTPL / MAE. Quito: Integraf. 2011

13 Neill C, Cerri C, Melillo J, Feigl B, Steudler P, Moraes J, Piccolo M. Las acciones y la dinámica de suelos de carbono después de la deforestación para pastos en Rondonia. En: Los procesos del suelo y el ciclo del carbono. La ciencia del suelo; Boca Raton. Florida: Lal R, Kimble J, Follett R, Stewart B (editores); 1998. pp. 9-28. 